\title{
meso and racemo Additions in Propagation for Radical Polymerization of Dialkyl Fumarates II. Determination of the Absolute Rate Constants
}

\author{
Masahiro Yoshioka, Akikazu Matsumoto, and Takayuki Orsu* \\ Department of Applied Chemistry, Faculty of Engineering, Osaka City University, \\ Sugimoto, Sumiyoshi-ku, Osaka 558, Japan
}

(Received March 14, 1991)

\begin{abstract}
The propagation process in radical polymerization of dialkyl fumarates (DRF) was investigated. The propagation rate constants $\left(k_{\mathrm{p}}\right)$ for some DRFs were determined by ESR spectroscopy. It was revealed that $k_{\mathrm{p}}$ increased with the introduction of a bulky tert-butyl ester group in the following order: di-tert-butyl fumarate $>$ methyl tert-butyl fumarate $>$ dimethyl fumarate. The absolute rate constants for meso and racemo additions were elucidated by the combination of the $k_{\mathrm{p}}$ and the probability of meso addition, which was determined by ${ }^{13} \mathrm{C}$ NMR spectroscopy.
\end{abstract}

KEY WORDS Dialkyl Fumarate / Radical Polymerization / Electron Spin Resonance / Propagation Rate Constant / meso Addition / racemo Addition /

Dialkyl fumarates (DRF) with bulky ester alkyl groups homopolymerize readily to give high molecular weight poly(substituted methylene)s (Scheme 1). ${ }^{1-5}$ We have revealed that the polymerization reactivity of DRF increases in the following order: di-tert-butyl fumarate $($ DtBF $)>$ diisopropyl fumarate $($ DiPF $) \approx$ methyl tert-butyl fumarate $(\mathrm{MtBF})>$ dimethyl fumarate (DMF). This order is consistent with the increase in the bulkiness of the ester alkyl groups, and has been interpreted as consequence of the decrease in a rate of bimolecular termination between less-flexible poly(DRF) radicals, relative to a rate of propagation. ${ }^{4,5}$

Rate constants for propagation $\left(k_{\mathrm{p}}\right)$ and termination $\left(k_{\mathrm{t}}\right)$ are popularly determined by the analysis of non-steady state by means of a rotating sector. ${ }^{6}$ Recently, however, it has been

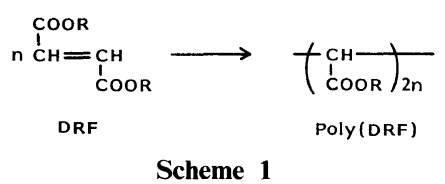

Scheme 1 demonstrated that ESR spectroscopy is also an another powerful tool for elucidation of the rate constants at non-steady and steady states. $^{7-12}$ In polymerization of DRF, the propagating poly(DRF) radicals are detectable easily under usual polymerization conditions by ESR without any special technique and apparatus, ${ }^{4,13-15}$ leading to the facile determination of the rate constants. For example, we have already determined the $k_{\mathrm{p}}$ and $k_{\mathrm{t}}$ for polymerizations of isopropyl tert-butyl fumarate initiated with dimethyl 2,2'-azobisisobutyrate (MAIB) or 2,2'-azobisisobutyronitrile by use of the ESR method. ${ }^{12}$ Furthermore, recent results indicate that $k_{\mathrm{p}}$ of DiPF is considerably larger than that of diethyl fumarate (DEF), ${ }^{13-15}$ suggesting that an increase in the propagation rate contributes to overall polymerization reactivity as well as retardation of termination by the introduction of bulky substituents.

In the preceding paper, ${ }^{16}$ we have determined the probabilities of meso and racemo additions, $P_{m}$ and $P_{r}$, respectively, in propagation 
of DRF by means of restricted trans mode opening of the double bond, and revealed the dependence of the $P_{m}$ on polymerization temperature by using ${ }^{13} \mathrm{C}$ NMR spectroscopy.

In this study, to obtain the information of propagation mechanisms for polymerization of DRFs, the $k_{\mathrm{p}}$ values for polymerization of DMF, MtBF and DtBF are determined by the ESR method, and the absolute values of $k_{m}$ and $k_{r}$ are evaluated from the combination of the $k_{\mathrm{p}}$ and $P_{m}$.

\section{EXPERIMENTAL}

DRFs were prepared and purified according to the methods previously reported. ${ }^{1-4} \mathrm{Com}$ mercial MAIB as a radical initiator was used after recrystallization from ethanol. 1,3,5Triphenylverdazyl was synthesized and purified as described in the literature. ${ }^{17}$

Polymerization was carried out in a sealed glass tube, similarly to the method described in the preceding paper. ${ }^{16}$

The measurement of ESR spectra was carried out by the use of a Bruker ESP-300 spectrometer with a variable temperature control. The operating conditions of ESR spectra were as follows; microwave frequency, $9.49 \mathrm{GHz}$; modulation frequency, $100 \mathrm{kHz}$; modulation amplitude, $0.1 \mathrm{G}$; time constant, $655.36 \mathrm{~ms}$.

\section{RESULTS AND DISCUSSION}

Figure 1 shows the time-conversion relationships for radical polymerization of DMF, MtBF and DtBF in benzene with MAIB at $60^{\circ} \mathrm{C}$. The rates of polymerization $\left(R_{\mathrm{p}}\right)$ calculated from the slopes of the curves are listed in Table $\mathrm{I}$, indicating that $R_{\mathrm{p}}$ increases in a following order; DtBF $>\mathrm{MtBF}>\mathrm{DMF}$, agreeing with the previous reports. ${ }^{4}$

The propagating radical of each polymerization system was observed directly by ESR spectroscopy. The shapes of the ESR spectra obtained for the polymerization of
DMF, MtBF and DtBF (Figure 2) are consistent with that of other DRFs reported previously, ${ }^{4,13-15}$ indicating that the ESR

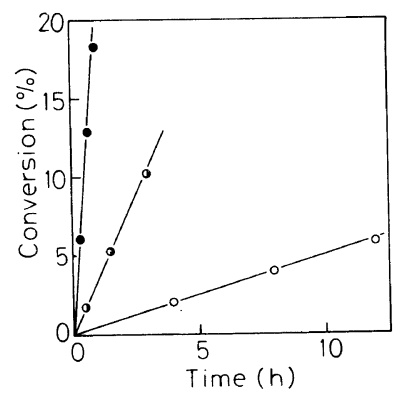

Figure 1. Time-conversion relationships for radical polymerization of $\operatorname{DMF}(\bigcirc), \operatorname{MtBF}(\circlearrowleft)$, and $\operatorname{DtBF}(\bullet)$ in benzene with $\mathrm{MAIB}$ at $60^{\circ} \mathrm{C}:[\mathrm{DRF}]=1.0 \mathrm{moll}^{-1}$; [MAIB] $=0.02 \mathrm{moll}^{-1}$

Table I. Determination of propagation rate constant $\left(k_{\mathrm{p}}\right)$ for radical polymerization of DRF at $60^{\circ} \mathrm{C}^{\mathrm{a}}$

\begin{tabular}{|c|c|c|c|}
\hline \multirow[t]{2}{*}{ DRF } & $R_{\mathrm{p}} \times 10^{5}$ & {$[\mathrm{M} \cdot] \times 10^{4}$} & $k_{\mathrm{p}}$ \\
\hline & $\mathrm{moll}^{-1} \mathrm{~s}^{-1}$ & $\operatorname{moll}^{-1}$ & $1 \mathrm{~mol}^{-1} \mathrm{~s}^{-1}$ \\
\hline DtBF & 5.14 & 1.11 & 0.46 \\
\hline $\mathrm{MtBF}$ & 0.95 & 0.41 & 0.23 \\
\hline DMF & 0.14 & 0.24 & 0.058 \\
\hline
\end{tabular}

${ }^{\mathrm{a}}[\mathrm{DRF}]=1.0 \mathrm{moll}^{-1} ;[\mathrm{MAIB}]=0.02 \mathrm{moll}^{-1}$ in benzene.

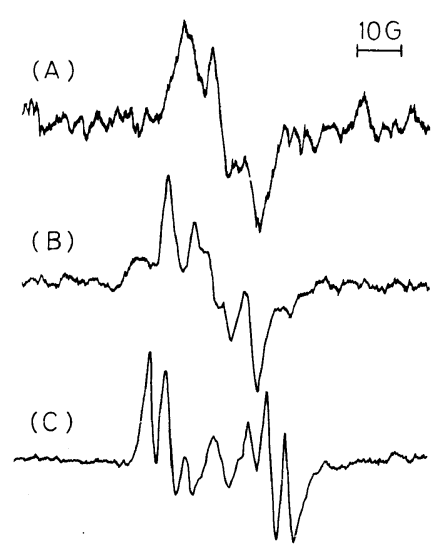

Figure 2. ESR spectra observed during radical polymerization of (A) DMF, (B) MtBF, and (C) DtBF in benzene with MAIB at $60^{\circ} \mathrm{C}:[\mathrm{DRF}]=1.0 \mathrm{moll}^{-1}$; $[\mathrm{MAIB}]=0.02 \mathrm{moll}^{-1}$. 
spectra arise from the propagating radicals. The absolute propagating radical concentration $([\mathrm{M} \cdot])$ was measured from the intensity of these spectra in a steady state by the use of the verdazyl as a standard, ${ }^{12}$ as shown in Table I. Table I also includes the $k_{\mathrm{p}}$ values for these DRFs, which were calculated from the $R_{\mathrm{p}}$ and [M•] according to eq 1 .

$$
k_{\mathrm{p}}=R_{\mathrm{p}} /[\mathrm{M} \cdot][\mathrm{DRF}]
$$

The obtained $k_{\mathrm{p}}$ values are much smaller than those for polymerization of ordinary vinyl monomers, and are in agreement with the $k_{\mathrm{p}}$ for other DRFs $\left(k_{\mathrm{p}}\left(30^{\circ} \mathrm{C}\right)=0.015\right.$ and 0.31 $1 \mathrm{~mol}^{-1} \mathrm{~s}^{-1}$ for DEF ${ }^{14}$ and DiPF, ${ }^{15}$ respectively). Moreover, it is clear that the $k_{\mathrm{p}}$ obtained increases with the increase of $R_{\mathrm{p}}$, i.e., both the $k_{\mathrm{p}}$ and $R_{\mathrm{p}}$ increase in the order of DtBF $>$ MtBF $>$ DMF. However, the $R_{\mathrm{p}}$ for DtBF was 36.7 times larger than that for DMF, while the $k_{\mathrm{p}}$ for DtBF was only 7.7 times larger, indicating that not only the increase in the $k_{\mathrm{p}}$ but also the decrease in $k_{\mathrm{t}}$ contribute to the higher polymerization reactivities of DRF bearing a bulky tert-butyl group.

The increase in $k_{\mathrm{p}}$ by introduction of the bulky ester groups is likely to contradict the steric hindrance in propagation. It was considered that a propagating manner of these DRFs should be clarified and discussed in order to interpret the increase of $k_{\mathrm{p}}$.

In the preceding paper, ${ }^{16}$ the differences in free energies of activation for meso and racemo additions $\left(\Delta G_{m}^{\neq}-\Delta G_{r}^{\ddagger}\right)$ at $60^{\circ} \mathrm{C}$ were determined, from which $P_{m}$ values at $60^{\circ} \mathrm{C}$ were calculated to be $0.73,0.57$ and 0.47 for DtBF, MtBF and DMF, respectively (Table II).

The $P_{m}$ obtained can be correlated to the rate constants for meso and racemo additions ( $k_{m}$ and $k_{r}$, respectively) in propagation (eq 2). ${ }^{18}$

$$
P_{m} / P_{r}=k_{m} / k_{r}
$$

Because the $k_{\mathrm{p}}$ may be expressed as eq 3 , the absolute $k_{m}$ and $k_{r}$ values can be obtained by
Table II. Probability of meso addition and absolute rate constants for meso and racemo additions in radical polymerization of DRF at $60^{\circ} \mathrm{C}$

\begin{tabular}{lcccc}
\hline DRF & $P_{m}^{\mathrm{a}}$ & \multicolumn{1}{c}{$k_{m}^{\mathrm{b}}$} & & \multicolumn{1}{c}{$k_{r}^{\mathrm{b}}$} \\
\cline { 5 - 5 } & & $1 \mathrm{~mol}^{-1} \mathrm{~s}^{-1}$ & & $1 \mathrm{~mol}^{-1} \mathrm{~s}^{-1}$ \\
\hline DtBF & 0.73 & 0.55 & & 0.21 \\
MtBF & 0.57 & 0.26 & & 0.19 \\
DMF & 0.47 & 0.054 & & 0.061 \\
\hline
\end{tabular}

a Calculated from $\Delta G_{m}^{\neq}-\Delta G_{r}^{\neq}$at $60^{\circ} \mathrm{C}$ (see ref 16).

b Calculated from eq 2 and 3 using $k_{\mathrm{p}}$ and $P_{m}$ (see the text).

the combination of the $P_{m}$ and $k_{\mathrm{p}}$ values.

$$
k_{\mathrm{p}}=P_{m} k_{m}+P_{r} k_{r}
$$

The $k_{m}$ and $k_{r}$ obtained for polymerization of DMF, MtBF and DtBF at $60^{\circ} \mathrm{C}$ are listed in Table II. The both $k_{m}$ and $k_{r}$ increased with the introduction of tert-butyl group as substituent, but the magnitude of increase in $k_{m}$ (from 0.054 for DMF to 0.55 for DtBF) was larger than that in $k_{r}$ (from 0.061 for DMF to 0.21 for DtBF). Therefore, it is concluded that the larger $k_{\mathrm{p}}$ for DtBF originates mainly from the increase in the value of $k_{m}$.

In the radical polymerization of ordinary vinyl monomers, since the stereochemical structure of the penultimate unit is determined by the addition of the attacking monomer to the propagating radical, the direction of addition and the opening mode of carbon-tocarbon double bond cannot be distinguished each other. On the other hand, in the case of polymerization of DRF, the direction of addition dominates the configuration of the bond which is newly formed by the addition, i.e., $P_{m}$. The $k_{m}$ and $k_{r}$ values determined in this work mean the preference of meso and racemo additions, respectively.

In conclusion, the $k_{\mathrm{p}}$ for polymerization of DtBF, MtBF and DMF at $60^{\circ} \mathrm{C}$ was determined from the concentration of the propagating radical by the ESR method, indicating that the high polymerization reactivity of 
DRF having a tert-butyl group was induced from both the increase in $k_{\mathrm{p}}$ and the decrease in $k_{\mathrm{t}}$. Moreover, the absolute rate constants for meso and racemo additions, $k_{m}$ and $k_{r}$, which are obtained from the combination of $k_{\mathrm{p}}$ and $P_{m}$, increase (especially in the case of $k_{m}$ ) by the introduction of a tert-butyl group into ester substituents, leading to the highest $k_{\mathrm{p}}$ and $P_{m}$ values of DtBF.

\section{REFERENCES AND NOTES}

1. N. Toyoda and T. Otsu, J. Macromol. Sci-Chem., A19, 1011 (1983).

2. T. Otsu and N. Toyoda, Polym. Bull., 11, 453 (1984).

3: T. Otsu, T. Yasuhara, K. Shiraishi, and S. Mori, Polym. Bull., 12, 449 (1984).

4. T. Otsu, T. Yasuhara, and A. Matsumoto, $J$. Macromol. Sci.-Chem., A25, 537 (1988).

5. A. Matsumoto, T. Tarui, and T. Otsu, Macromolecules, 23, 5102 (1990).

6. K. C. Berger and G. Meyerhoff, "Polymer Handbook," 3rd ed, J. Brandrup and E. H. Immergut, Ed., Wiley, New York, N. Y., 1989, p II-67.

7. S. E. Bresler, E. N. Kazbekov, V. N. Fomichev, and
V. N. Shadrin, Makromol. Chem., 157, 167 (1972).

8. M. Kamachi, M. Kohno, Y. Kuwae, and S. Nozakura, Polym. J., 14, 749 (1982); M. Kamachi, Y. Kuwae, and S. Nozakura, ibid., 17, 541 (1985).

9. T. Sato, S. Inui, H. Tanaka, T. Ota, M. Kamachi, and K. Tanaka, J. Polym. Sci., Polym. Chem. Ed., 25, 637 (1987).

10. R. W. Garrett, D. J. T. Hill, J. H. O’Donnell, P. J. Pomery, and C. L. Winzor, Polym. Bull., 22, 611 (1989).

11. S. Zhu, S. Tian, and A. E. Hamielec, Macromolecules, 23, 1144 (1989).

12. M. Yoshioka and T. Otsu, Polym. Prepr. Jpn., 39, 1697 (1990); Macromolecules, submitted.

13. T. Otsu, Makromol. Chem., Macromol. Symp., 10/11, 235 (1987).

14. T, Otsu, B. Yamada, and T. Ishikawa, Macromolecules, 24, 415 (1991).

15. B. Yamada, E. Yoshikawa, H. Miura, K. Shiraishi, and T. Otsu, Polymer, 32, 1892 (1991).

16. M. Yoshioka, A. Matsumoto, and T. Otsu, Polym. J., 23, 1191 (1991).

17. R. Kuhn and H. Trischmann, Monat. Chem., 95, 457 (1964).

18. Since there are possibilities of head-to-head or tailto-tail propagation as well as head-to-tail in the case of $\mathrm{MtBF}, P_{m}, k_{\mathrm{p}}$ and $k_{m}$ determined in the present and preceding papers mean apparent values. 